\title{
Maximum Diameter of Native Abdominal Aortic Aneurysm Measured by Angio-Computed Tomography
}

\section{Reproducibility and Lack of Consensus Impacts on Clinical Decisions}

\author{
Caroline E. Mora, MD' ${ }^{1}$, Claude D. Marcus, MD¹, Coralie M. Barbe, MD², Fiona B. Ecarnot, MSc ${ }^{3}$, \\ Anne L. Long, MD ${ }^{4,5, *}$ \\ ${ }^{1}$ Department of Radiology, University Hospital Reims, Hôpital Robert Debré, Reims, France \\ ${ }^{2}$ Clinical Research Unit, University Hospital Reims, Hôpital Robert Debré, Reims, France \\ ${ }^{3}$ EA3920, Department of Cardiology, University Hospital Besancon, Besançon, France \\ ${ }^{4}$ Department of Internal Medicine and Vascular Medicine, Pavillon M, Hospices Civils de Lyon, University Hospital Edouard Herriot, \\ Lyon, France \\ ${ }^{5}$ Faculty of Medicine and Maieutic Charles Merieux, Claude Bernard Lyon 1 University, Oullins, France
}

\begin{abstract}
Background: Computed tomography angiography (CTA) is the reference technique for the measurement of native maximum abdominal aortic aneurysm (AAA) diameter when surgery is being considered. However, there is a wide choice available for the methodology of maximum AAA diameter measurement on CTA, and to date, no consensus has been reached on which method is best. We analyzed clinical decisions based on these various measures of native maximum AAA diameter with CTA, then analyzed their reproducibility and identified the method of measurement yielding the highest agreement in terms of patient management.

Materials and Methods: Three sets of measures in 46 native AAA were obtained, double-blind by three radiologists $(J, S, V)$ on orthogonal planes, curved multiplanar reconstructions, and semi-automated-software, based on the AAA-Iumen centerline. From each set, the clinical decision was recorded as follows: "Follow-up" (if all diameters $<50 \mathbf{~ m m}$ ), "ambiguous" (if at least one diameter $<50 \mathrm{~mm}$ AND at least one $\geq 50 \mathrm{~mm}$ ) or "Surgery " (if all diameters $\geq 50 \mathrm{~mm}$ ). Intra- and interobserver agreements in clinical decisions were compared using the weighted Kappa coefficient.
\end{abstract}

Results: Clinical decisions varied according to the measurement sets used by each observer, and according to intra and interobserver (lecture\#1) reproducibility. Based on the first reading of each observer, the number of AAA proposed for surgery ranged from 11 to 24 for J, 5 to 20 for $S$, and 15 to 23 for V. The rate of AAAs classified as "ambiguous" varied from $11 \%(5 / 46)$ to $37 \%(17 / 46)$.

The semi-automated method yielded very good intraand interobserver agreements in clinical decisions in all comparisons (Kappa range 0.83-1.00).

Conclusion: The semi-automated method seems to be appropriate for native AAA maximum diameter measurement on CTA. In the absence of AAA outer-wallbased software more robust for complex AAA, clinical decisions might best be made with diameter values obtained using this technique.

Copyright $\odot 2015$ Science International Corp.

\section{Key Words}

Abdominal aortic aneurysm - Computed tomography • Reproducibility

\section{* Corresponding Author:}

Anne Long, MD

Department of Internal Medicine and Vascular Medicine

Groupement Hospitalier Edouard

Herriot, Place d'Arsonval, 69437 Lyon Cedex 03, France

Phone: +33 4721110 49; Fax: +33 4721175 67; E-mail: anne.long@chu-lyon.fr 


\section{Introduction}

The maximum diameter of native abdominal aortic aneurysm (AAA) is the main parameter used to monitor patient care, since it is related to the risk of rupture. Surgery is recommended in men when the maximum diameter is 50 to $55 \mathrm{~mm}$ (depending on the country or when patients are at higher risk of rupture), or when AAA diameter grows by more than 10 $\mathrm{mm}$ per year [1-3]. Computed tomography angiography (CTA) is the reference technique for the measurement of AAA diameter when surgery is being considered. However, there is a wide choice available for the methodology of maximum AAA diameter measurement on CTA, and to date, no consensus has been reached on which method is best [4,5].

A gold standard imaging technique should have a high reproducibility rate. Previous studies of the reproducibility of diameter measurement by CTA have generally been based on analysis of the difference between diameter values. The most popular statistical method is the Bland and Altman method [6]. When applied to AAA diameter analysis, the clinical threshold is $5 \mathrm{~mm}$ for the coefficient of repeatability, and the clinically accepted range is $-5 \mathrm{~mm}$ to $+5 \mathrm{~mm}$ for the limits of agreement [7,8]. Mora et al. [5] recently showed that even using precise methodology, the reproducibility of maximum diameter measurements of native AAA on CTA may not meet recommended thresholds.

In the end, the clinical decision regarding management is based on a maximum diameter value that is measured in a non-consensual fashion, which may furthermore differ from one observer to another due to lack of reproducibility. The impact of various CTAbased measures of maximum AAA diameter, and the impact of reproducibility limits on the decision to operate have never been investigated.

Therefore, the aims of this study were to analyze the consequences on clinical decisions of such a wide choice of AAA maximum diameter measurement methodologies when using CTA; to analyze the reproducibility of these clinical decisions, and finally, to identify the method of measurement that yields the best agreement for patient management.

\section{Materials and Methods}

The details of CTA protocol, database constitution, analysis and patient selection have previously been described [5]. CTA acquisitions were not ECG (electrocardiogram) gated.

Male patients with non-operated infrarenal AAA undergoing CTA between January 1, 2010 and April 15, 2012 were retrospectively identified in the Picture Archiving and Communication System (PACS) (Impax version 5.2; Agfa, Mortsel, Belgium) of the Radiology Department of the University Hospital of Reims, France.

Patients were identified using the terms "aneurysm," "aorta," "abdominal," and "male gender" and combined using the Boolean operators "AND" and "OR," from the computerized indication for CTA examination in the PACS. After reading the whole indication for CTA examination, patients were excluded in case of inflammatory aneurysm, false aneurysm, aneurysm after open repair or stent-graft, thoracic, thoraco-abdominal or iliac artery aneurysms, or aortic diameter less than $30 \mathrm{~mm}$. Older CTA were selected in case of multiple examinations for the same patient. CTA from eligible patients were re-analyzed on axial slices and only CTAs at the arterial phase after contrast injection showing an infrarenal AAA with a maximum external diameter in any direction greater than or equal to $30 \mathrm{~mm}$ were identified [5]. Only patients with an AAA greater than or equal to $40 \mathrm{~mm}$ and less than or equal to $60 \mathrm{~mm}$ were included in the present analysis. Age was the only patient characteristic that was recorded.

One junior resident $(J)$, one senior vascular interventional radiologist (S) with, respectively, 3 and 20 years experience, and a specialist in vascular medicine with more than 15 years experience in vascular radiology (V), all blinded to previous radiological reports, independently measured maximum AAA diameter on each examination. Each observer performed two readings, at a minimum of 4 week intervals, yielding 6 series of measures: Junior readings $1(\mathrm{~J} 1)$ and $2(\mathrm{~J} 2)$, Senior readings 1 (S1) and 2 (S2), and Vascular readings 1 (V1) and 2 (V2).

Maximum external diameter was measured using 10 different methodologies. The slices of interest displaying the largest aneurysm diameter were selected by each observer ( $\mathrm{J}, \mathrm{S}$, and $V$ ), although the slice numbers were not recorded.

First, nine measurements were performed on the PACS workstation:

1. On the selected axial slice (Figure $1 \mathrm{~A}$ ):

- antero-posterior diameter Axial_APD,

- transverse diameter Axial_TrD,

- maximum diameter in any direction Axial_Dmax.

2. On the selected sagittal and coronal multiplanar reconstruction (MPR) images:

- on sagittal MPR (Figure 1B), antero-posterior diameter Sag_APD and diameter perpendicular to the long axis of the aneurysm Sag_PerpD,

- on coronal MPR (Figure 1C), transverse diameter Coro_TrD, and diameter perpendicular to the long axis of the aneurysm Coro_PerpD,

3. Using dedicated software (3D Voxar 6.3.2 Workstation, Toshiba Medical Visualization System Europe Ltd, Edinburgh, UK) providing curvilinear MPR: 

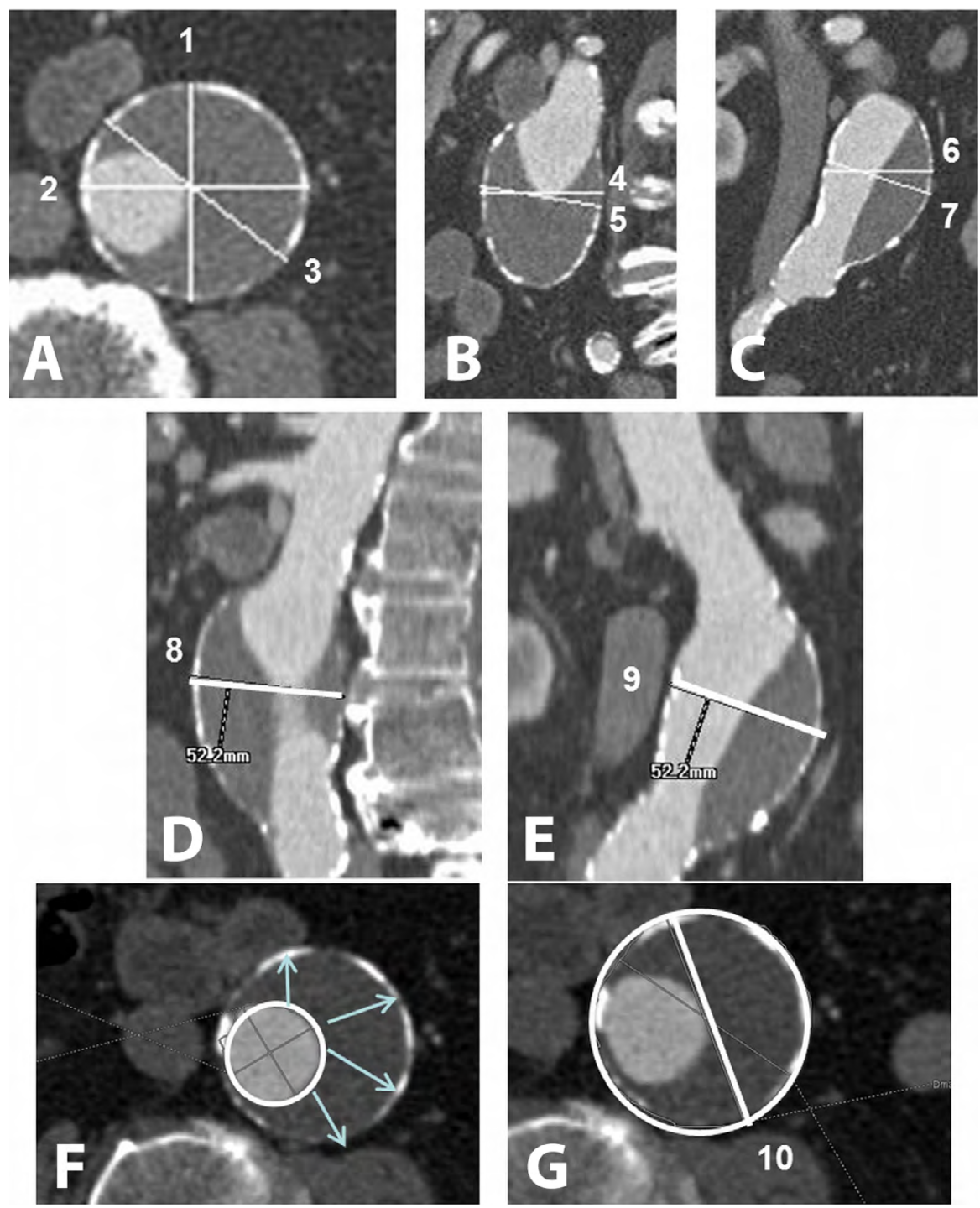

Figure 1. Diameters measured on native axial slices (upper left), sagittal and coronal planes (upper middle and right), on parasagittal, paracoronal curvilinear multiplanar reconstruction (MPR) (middle right and left), and with the semi-automated method (lower right and left). A. Axial slices: antero-posterior diameter Axial_APD (1); transverse diameter Axial_TrD (2); and maximum diameter in any direction Axial_Dmax (3). B. Sagittal MPR image: antero-posterior diameter Sag_APD (4) and diameter perpendicular to the long axis of the aneurysm Sag_PerpD (5). C. Coronal MPR image: transverse diameter Coro_TrD (6) and diameter perpendicular to the long axis of the aneurysm Coro_PerpD (7). D. Parasagittal reconstructed image: antero-posterior diameter perpendicular to the long axis of the aneurysm PSR_PerpD (8). E. Paracoronal reconstructed images: transverse diameter perpendicular to the long axis of the aneurysm PCR_PerpD (9). F. Semi-automated method: cross section containing the maximum aortic diameter in any direction perpendicular to the lumen centerline. The circle corresponds to the initial result obtained with the software, contouring the interface between the aortic lumen opacified by contrast agent and the thrombus. Arrows illustrate the effect of manual drawing of the AAA outer limits (thrombus and walls) performed by the observer. G. Semi-automated method: maximum diameter in any direction (10) automatically calculated once the observer has manually drawn the outer limits of the AAA, including thrombus and the arterial wall

- on selected parasagittal reformatted images (Figure 1D), antero-posterior diameter perpendicular to the long axis of the aneurysm PSR_PerpD,

- on selected paracoronal reformatted images (Figure 1E), transverse diameter perpendicular to the long axis of the aneurysm PCR_PerpD.

Second, using dedicated 3D analysis software (Advanced Vessel Analysis Xpress, General Electric, Milwaukee, WI, USA), 
Table 1. Comparison between abdominal aortic aneurysm (AAA) diameter values obtained from the first reading of each observer.

\begin{tabular}{llll}
\hline Diameter $^{*}$ & Reading J1 & Reading S1 & Reading V1 \\
\hline Axial_APD & $46.2 \pm 6.1$ & $46 \pm 5.8$ & $48 \pm 6.2$ \\
Axial_TrD & $47.5 \pm 5.6$ & $46.6 \pm 5.7$ & $48.3 \pm 5.7$ \\
Axial_Dmax & $51.5 \pm 5.1$ & $48.8 \pm 5.2$ & $51.1 \pm 5.2$ \\
Sag_APD & $47.3 \pm 5.7$ & $45.3 \pm 6$ & $48 \pm 6$ \\
Sag_PerpD & $47.3 \pm 5.5$ & $45 \pm 5.7$ & $48 \pm 6.1$ \\
Coro_TrD & $47.9 \pm 5.9$ & $46 \pm 5.6$ & $48.4 \pm 5.9$ \\
Coro_PerpD & $47.5 \pm 5.7$ & $44.7 \pm 5.7$ & $47.7 \pm 5.6$ \\
PSR_PerpD & $47 \pm 5.9$ & $45.1 \pm 5.6$ & $47.8 \pm 5.7$ \\
PCR_PerpD & $47.5 \pm 5.5$ & $45.5 \pm 5.4$ & $48.2 \pm 5.9$ \\
Semi-auto- & $49.9 \pm 5.3$ & $48.9 \pm 5.4$ & $49.7 \pm 5.9$ \\
mated_D & & & \\
P & 0.0004 & 0.0006 & 0.125 \\
\hline
\end{tabular}

Observers: $\mathrm{J1}, \mathrm{S1}, \mathrm{V} 1 ; \mathrm{n}=46 \mathrm{AAA}$; diameters are expressed in $\mathrm{mm}$ as the mean \pm standard deviation; " for diameter abbreviations, see Figure 1; PSR/ PCR: parasagittal/paracoronal reconstruction.

the maximum diameter perpendicular to the centerline called Semi-automated_D was measured semi-automatically (Figure $1 \mathrm{~F}$ and Figure $1 \mathrm{G})$. This $3 \mathrm{D}$ analysis software creates the abdominal aortic lumen centerline once the observer has placed two points at the celiac aortic level and the aortic bifurcation, and then the software automatically provides cross sections perpendicular to this centerline. The cross-section containing the maximum aortic diameter in any direction perpendicular to the lumen centerline is visually selected by the observer, who then manually draws the outer limits of the AAA, including thrombus and the arterial wall. Then, the maximum diameter in any direction is automatically calculated.

The main difference between the two methods is that the centerline of MPR curvilinear is drawn manually when it is drawn automatically with the semi-automated method from the starting point to the ending point of the aorta.

The study was approved by the Institutional Review Board of Reims University Hospital, France.

Data are described as mean and standard deviation (SD) for quantitative variables and number (percentage) for qualitative variables.

For each AAA and for each reading, the diameter values were classified into three different sets as follows:

- Orthogonal plane diameters (seven diameters)

- Curvilinear MPR diameters (two diameters)

- Semi-automated method (one diameter)

When analyzing values obtained for a single AAA in any one of the sets, the clinical decisions were recorded as follows:

- "Follow-up" if all diameters were less than $50 \mathrm{~mm}$,

- "Ambiguous" if at least one diameter was less than $50 \mathrm{~mm}$
Table 2. Clinical decisions based on three sets of abdominal aortic aneurysm (AAA) maximum diameter values, for the first and second readings of each observer.

\begin{tabular}{lllllll}
\hline Clinical decision & J1 & J2 & S1 & S2 & V1 & V2 \\
\hline
\end{tabular}

Orthogonal Plane Diameters

$\begin{array}{lllllll}\text { Follow up (n) } & 18 & 16 & 24 & 24 & 18 & 19 \\ \text { Ambiguous (n) } & 17 & 15 & 17 & 13 & 13 & 12 \\ \text { Intervention (n) } & 11 & 15 & 5 & 9 & 15 & 15\end{array}$

Curvilinear MPR Diameters

$\begin{array}{lllllll}\text { Follow up (n) } & 24 & 26 & 31 & 29 & 23 & 22 \\ \text { Ambiguous (n) } & 7 & 5 & 7 & 8 & 5 & 5 \\ \text { Intervention (n) } & 15 & 15 & 8 & 9 & 18 & 19 \\ \text { Semi-Automated Method } \\ \text { Follow up (n) } & 22 & 23 & 26 & 26 & 23 & 23 \\ \text { Intervention (n) } & 24 & 23 & 20 & 20 & 23 & 23\end{array}$

$n=46$ AAA; for each AAA, the clinical decision was established separately based on seven diameters measured for the "orthogonal planes" set, based on two diameters for the "curvilinear MPR diameters" set, and based on only one diameter with the semi-automated method.

and at least one other was greater than or equal to $50 \mathrm{~mm}$ - "Intervention" if all diameters were greater than or equal to $50 \mathrm{~mm}$

As only one diameter was provided with the semi-automated method, the clinical decision was recorded as "Follow-up" in case of AAA less than $50 \mathrm{~mm}$ or "Intervention" in case of AAA greater than or equal to $50 \mathrm{~mm}$.

For each reader, mean diameters obtained with each method were compared with the general linear model and the Student $t$ test.

Proportions of "Intervention" decisions were compared between the two sets of measures of each individual observer $(\mathrm{J} 1, \mathrm{~S} 1$, and $\mathrm{V} 1)$ and between the same set of measures of two observers ( $\mathrm{J} 1$ versus $\mathrm{S} 1$, J1 versus $\mathrm{V} 1$, and $\mathrm{S} 1$ versus $\mathrm{V} 1$ ) using Mac Nemar's Chi test.

Intra-observer (J1 versus J2; S1 versus S2, and V1 versus V2) and inter-observer agreement in terms of clinical decisions were compared using the Kappa coefficient $(\mathrm{K})$. The weighted Kappa coefficient was used when clinical decisions were "Follow-up," "Ambiguous," or "Intervention." Ordinary (non-weighted) Kappa coefficient was used when the clinical decision was a binary variable ("Follow-up" or "Intervention"), that is, for the semi-automated method. The strength of agreement was interpreted as very good when $\mathrm{K}$ was greater than 0.81 , good when $\mathrm{K}$ was $0.61-0.80$, moderate when $\mathrm{K}$ was $0.41-0.60$, fair when $\mathrm{K}$ was $0.21-0.40$, poor when $\mathrm{K}$ was less than 0.20 .

A P value less than 0.05 was considered statistically significant. All analyses were performed using SAS version 9.0 (SAS Inc, Cary, NC, USA). 
Table 3. $P$ values from intra- and inter-observer comparisons of the number of patients proposed for intervention, based on each set of measures.

\begin{tabular}{|c|c|c|c|}
\hline $\begin{array}{l}\text { Intra-observer } \\
\text { comparison }\end{array}$ & J1 & S1 & V1 \\
\hline $\begin{array}{l}\text { Orthogonal plane } \\
\text { diameters vs. curvilinear } \\
\text { MPR diameters }\end{array}$ & 0.04 & 0.08 & 0.08 \\
\hline $\begin{array}{l}\text { Orthogonal plane } \\
\text { diameters vs. semi- } \\
\text { automated method }\end{array}$ & 0.0003 & 0.0001 & 0.005 \\
\hline $\begin{array}{l}\text { Curvilinear MPR diameters } \\
\text { vs. semi-automated } \\
\text { method }\end{array}$ & 0.003 & 0.0005 & 0.02 \\
\hline $\begin{array}{l}\text { Inter-observer } \\
\text { comparison }\end{array}$ & J1 vs. S1 & J1 vs. V1 & S1 vs. V1 \\
\hline $\begin{array}{l}\text { Orthogonal plane } \\
\text { diameters }\end{array}$ & 0.03 & 0.04 & 0.002 \\
\hline $\begin{array}{l}\text { Curvilinear MPR } \\
\text { diameters }\end{array}$ & 0.008 & 0.08 & 0.002 \\
\hline Semi-automated method & 0.04 & 0.31 & 0.08 \\
\hline
\end{tabular}

Intra-observer: between two sets of diameters of one observer, from the first reading; Inter-observer: between the same set of all observers, from the first reading.

\section{Results}

In total, 46 patients (corresponding to 46 CTA) were included in the present study. The mean age was 71 \pm 9 years.

The comparisons of the 10 mean diameters noted by each observer on the first reading are reported in Table 1.

For readers $\mathrm{J}$ and $\mathrm{S}$, there was a significant difference overall in mean diameters obtained with each method $(P=0.0004$ and $P=0.0006$ respectively). For reader $\mathrm{J}$, the diameters that differed significantly were Axial_APD $(P=0.03)$, Axial_Dmax $(P<0.0001)$ and diameter measured by the semi-automated method $(P=0.02)$. For reader $S$, the diameters that differed significantly were Axial_Dmax $(P=0.001)$ and the semi-automated method $(P=0.0007)$.

\section{Clinical Decisions}

Clinical decisions based on the 3 sets of AAA maximum diameter values, from the first and second readings of each observer, are reported in Table 2 . The
Table 4. Intra-observer agreement in clinical decisions between the first and the second readings of each observer.

\begin{tabular}{|c|c|c|}
\hline $\begin{array}{l}\text { Intra-observer } \\
\text { agreement }\end{array}$ & $\begin{array}{l}\text { Agreement } \\
\text { n, \% }\end{array}$ & $\begin{array}{l}\text { Weighted Kappa } \\
\text { [range] }\end{array}$ \\
\hline \multicolumn{3}{|c|}{ Between both readings by junior (J1 vs. J2) } \\
\hline Orthogonal plane diameters & $40(87.0)$ & $0.85[0.74-0.96]$ \\
\hline Curvilinear MPR diameters & $44(95.7)$ & $0.95[0.89-1.00]$ \\
\hline Semi-automated method* & $43(93.5)$ & $0.87[0.73-1.00]$ \\
\hline \multicolumn{3}{|c|}{ Between both reading by senior (S1 vs. S2) } \\
\hline Orthogonal plane diameters & $42(91.3)$ & $0.89[0.78-0.99]$ \\
\hline Curvilinear MPR diameters & $39(84.8)$ & $0.80[0.66-0.94]$ \\
\hline Semi-automated method* & $46(100.0)$ & 1.00 \\
\hline \multicolumn{3}{|c|}{ Between both readings by vascular (V1 vs. V2) } \\
\hline Orthogonal plane diameters & $45(97.8)$ & $0.98[0.93-1.00]$ \\
\hline Curvilinear MPR diameters & $42(91.3)$ & $0.91[0.83-0.99]$ \\
\hline Semi-automated method* & $46(100.0)$ & 1.00 \\
\hline
\end{tabular}

clinical decision varied according to the sets used for each observer. The number of AAA proposed for "Intervention" ranged from 11 to 24 for J1, 5 to 20 for S1 and 15 to 23 for V1.

The clinical decision also varied between the first and second readings of a same observer, and between the first reading of 2 observers. The semi-automated method yielded the highest rate of AAA proposed for "Intervention".

The differences in the number of patients proposed for "Intervention" based on each set of measures (intra- and inter-observer comparisons) are reported in Table 3. From one set of measures to another, the decision to proceed to intervention differed significantly for each observer.

When the same set of measures by each observer was considered, the decision to proceed to intervention differed statistically significantly from one observer to another in a total of six of nine comparisons.

Based on the first reading of each observer, the rate of AAAs classified as "Ambiguous" varied from $11 \%(5 / 46)$ to $37 \%(17 / 46)$.

AAA Maximum Diameter on CT: Impact on Clinical Decisions 
Table 5. Inter-observer agreement in clinical decisions between the first reading of all observers.

\begin{tabular}{|c|c|c|}
\hline Interobserver Agreement & $\begin{array}{l}\text { Agreement } \\
\text { n (\%) }\end{array}$ & $\begin{array}{l}\text { Weighted Kappa } \\
\text { [range] }\end{array}$ \\
\hline \multicolumn{3}{|c|}{ Between first reading by junior and senior (J1 vs. S1) } \\
\hline Orthogonal plane diameters & $32(69.6)$ & $0.62[0.46-0.78]$ \\
\hline Curvilinear MPR diameters & $34(73.9)$ & $0.60[0.41-0.80]$ \\
\hline Semi-automated method* & $42(91.3)$ & $0.83[0.67-0.99]$ \\
\hline \multicolumn{3}{|c|}{ Between first reading by junior and vascular (J1 vs. V1) } \\
\hline Orthogonal plane diameters & $40(87.0)$ & $0.85[0.74-0.96]$ \\
\hline Curvilinear MPR diameters & $39(84.8)$ & $0.82[0.69-0.95]$ \\
\hline Semi-automated method ${ }^{*}$ & $45(97.8)$ & $0.96[0.87-1.00]$ \\
\hline \multicolumn{3}{|c|}{ Between first reading by senior and vascular (S1 vs. V1) } \\
\hline Orthogonal plane diameters & $30(65.2)$ & $0.60[0.45-0.75]$ \\
\hline Curvilinear MPR diameters & $33(71.7)$ & $0.58[0.39-0.77]$ \\
\hline Semi-automated method* & $43(93.5)$ & $0.87[0.73-1.00]$ \\
\hline
\end{tabular}

* Ordinary (non-weighted) Kappa coefficient, since there are only two outcomes, namely "Follow-up" or "Intervention".

\section{Intra-Observer Agreement in Clinical Decisions}

Intra-observer agreement in clinical decisions between the first and second readings of all observers is reported in Table 4. The highest rate of agreement was observed with Curvilinear MPR diameters for J $(\mathrm{K}=0.95)$, and with the semi-automated method for $\mathrm{S}$ and $\mathrm{V}(\mathrm{K}=1)$. The semi-automated method yielded 93.5\% agreement for $\mathrm{J}(\mathrm{K}=0.87)$.

\section{Interobserver Agreement in Clinical Decisions}

Inter-observer agreement in clinical decisions between the first reading of all observers is also reported in Table 5. The highest rates of agreement were observed with the semi-automated method, with a (non-weighted) Kappa coefficient ranging from 0.83 ( $\mathrm{J} 1$ versus $\mathrm{S} 1$ ) to 0.96 ( $\mathrm{J} 1$ versus $\mathrm{V} 1$ ).

\section{Discussion}

The aim of this study was to evaluate the impact on management of 46 patients with an AAA diameter between $40 \mathrm{~mm}$ and $60 \mathrm{~mm}$, of the lack of consensual methodology for the measurement of maximum diameter, and of the inadequate reproducibility of AAA maximum diameter measurement on CTA. We showed that clinical decisions varied according to the measurement sets used by each observer; that they also varied between the first and second reading of a same observer, and between first readings of two observers. The semi-automated method yielded good intra- and interobserver agreement in terms of clinical decisions in all comparisons.

Given that there is no consensus or recommendation in clinical practice for the preference of any single diameter over another, we chose to analyze the clinical decisions made using three different measurement sets, namely measures performed on orthogonal planes, from curvilinear multiplanar reconstructions and with the semi-automated method.

This analysis showed that clinical decisions differed for a same observer from one set of diameters to another, and also from one observer to another, even when the two observers were using the same set of diameters. The rate of patients referred to "Intervention" varied consistently.

The semi-automated method yielded the highest rates of surgical indication ( $\geq 50 \mathrm{~mm}$ ) for all observers, and for both readings $(24,20$, and $23 / 46$ patients for $\mathrm{J} 1, \mathrm{~S} 1$, and $\mathrm{V} 1$ respectively ; 23, 20, and 23/46 patients for J2, S2, and V2 respectively). This can likely be explained by the absence of an "ambiguous" option. Indeed, since only one diameter is obtained with the semi-automated method, only two clinical decisions are available, namely "Intervention" or "Follow-up".

Intra-observer agreement in clinical decisions was very good, with a Kappa coefficient greater than 0.81 for all sets of measurements, except one at 0.80 . The highest rate of agreement (100\%) was observed for observers $\mathrm{S}$ and $\mathrm{V}$ with the semi-automated method.

Inter-observer agreement in clinical decisions was poorer, as three of the kappa values dropped to 0.60 or below. The highest rates of agreement were observed for all three observers with the semi-automated method (91.3\% to $97.8 \%$, corresponding to a 
kappa coefficient ranging from 0.83 to 0.96 ), reflecting good strength of agreement.

These results should prompt a prudent attitude. Indeed, even when the Kappa coefficient is considered, in statistical terms, to be good or very good, closer analysis of the clinical decisions is mandatory to examine any discrepancies. For instance, the inter-observer agreement between $\mathrm{J} 1$ and S1 observed with the semi-automated method yielded a kappa coefficient of 0.83 , indicating very good agreement. Nonetheless, nearly $10 \%$ of patients (4/46) had contrasting management recommendations.

Previous studies have analyzed the reproducibility of various methodologies for AAA maximum diameter measurements in order to reach a consensus on the method that yields the highest reproducibility, but that is nonetheless available in routine clinical practice [5, 9-12]. The question of reproducibility is especially crucial when CTA images are analyzed by several different clinicians during the patient's management pathway.

To the best of our knowledge, this is the first study to report the impact of this multiplicity of measurement choices and their reproducibility on clinical decisions. Given that a difference of only a few millimeters may heavily impact on the choice of management strategy, the originality of this study lies in the determination of the type of measurement that gives the highest rate of agreement in terms of clinical decisions.

In a recent review of the Cochrane database, shortterm mortality (defined as 30-day or in-hospital mortality) after endovascular AAA repair and after open surgery were respectively $1.4 \%$ and $4.2 \%$ [13]. On the other hand, the rate of rupture of a $50 \mathrm{~mm}$-AAA per 1000 person-years was estimated at $6.4(95 \% \mathrm{Cl} 4.3-$ 9.5) in men in the RESCAN meta-analysis [14]. The recent IMPROVE trial showed that the 30-day mortality rate after rupture in patients was $35.4 \%$ in the endovascular strategy group and $37.4 \%$ in the open repair group [15]. Overall mortality is likely higher, as some patients die before reaching hospital. Therefore, the clinical decision in patients with AAA diameters close to the threshold has serious consequences for patient outcome, and a few millimeters can potentially influence the course of a patient's life.

The type of measurement and the reproducibility may only slightly affect intra- and inter-observer

Mora, C. et al. agreement in terms of clinical decisions when the AAA is small, that is, diameter less than $40 \mathrm{~mm}$, or when the AAA is clearly very big, that is, greater than $60 \mathrm{~mm}$. Even if there is a difference of $10 \mathrm{~mm}$ between two readings by the same observer or between two observers, the decision will still be "Follow-up" in the first case and "Intervention" in the second. However, for AAAs with a diameter close to the threshold value for Intervention $(50 \mathrm{~mm}$ ), in either direction (above or below), the impact of a few $\mathrm{mm}$ difference may be of greater importance. For this reason, we decided to limit our analysis to AAA with axial_Dmax ranging from $40 \mathrm{~mm}$ to $60 \mathrm{~mm}$, as being the zone where lack of reproducibility is most likely to tip the balance from one management strategy to the other. In addition, inclusion of smaller and bigger AAA might have interfered with the results, by respectively increasing the rates of "Follow-up" and "Intervention."

The semi-automated method provides a more realistic representation of AAA anatomy and consequently, the maximum diameter in any direction perpendicular to the lumen centerline estimated with this approach is closer to the real maximum diameter. However, in case of tortuous AAA with asymmetric thrombus, the Dmax measured perpendicular to the lumen centerline do not really represent the 'real' Dmax. A centerline based on the thrombus envelop (AAA outer-wall centerline) is in this case more adapted. Unfortunately, most of the software used with CT are based on the lumen centerline [16], even if some publications are based on AAA outer-wall centerline $[11,17,18]$. Software providing the AAA outer-wall centerline was not available in our Radiology Department.

With the semi-automated method, only one diameter value is provided for each AAA, leading to only two clinical outcomes, namely "Follow-up" or "Intervention". Therefore, the problematic clinical situation of an "Ambiguous" decision, leading to a decision to propose Intervention in a patient who in fact only requires Follow-up, or vice versa, is removed. A recent study showed that the semi-automated method led to more reproducible results between different observers [5]. Indeed, when using this method, human Intervention during the different steps of CT analysis is reduced, and is limited to the observer having to place two points at the celiac aortic level and the aortic bifurcation, choosing the slice of interest, then

AAA Maximum Diameter on CT: Impact on Clinical Decisions 
delimiting the outer wall of the aorta manually [5]. In the present study, the semi-automated method also yielded the highest rate of agreement in clinical decisions between observers, and between the first and second readings of two observers.

The study has some limitations that deserve to be acknowledged. Firstly, as the CTA protocol did not include cardiac gating, it is not possible to know whether measures were performed in the systolic or diastolic phase. However, this does not influence the results, since the same sets were read by all observers. Secondly, we did not record for each patient the presence or absence of wall calcifications, which might have helped identify external diameter. Similarly, we did not record the location (concentric or excentric) of any thrombus, which might have affected measurement with the semi-automated method. However, again, these points should not influence the results, since all observers read the same sets. Thirdly, we did not record AAA morphology; therefore, we could not discuss the impact of complex morphology on decisions agreements.

\section{Conclusion}

Even when AAA maximum diameter was measured on CTA considered as the gold standard, clinical decisions varied according to the measurement sets used by each observer, varied between readings by one observer and between the first readings of two observers. The semi-automated method, using lumen-based software, yielded very good intra- and inter-observer agreement in clinical decisions in all comparisons. These findings suggest that the semi-automated method seems to be appropriate for AAA maximum diameter measurement on CTA. In the absence of AAA outer-wall based software more robust for complex AAA, clinical decisions on whether or not to proceed to Intervention might reliably be made with diameter values obtained using this technique.

\section{Conflict of Interest}

The authors have no conflict of interest relevant to this publication.

\section{Comment on this Article or Ask a Question}

\section{References}

1. Brewster DC, Cronenwett JL, Hallett JW Jr, Johnston KW, Krupski WC, Matsumura JS. Joint Council of the American Association for Vascular Surgery and Society for Vascular Surgery. Guidelines for the treatment of abdominal aortic aneurysms. Report of a subcommittee of the Joint Council of the American Association for Vascular Surgery and Society for Vascular Surgery. J Vasc Surg. 2003;37:1106-1117. DOI: 10.1067/ mva.2003.363

2. Branchereau A. Small aortic aneurysm: Is evidence evident? Eur J Vasc Endovasc Surg. 2004;27:363-365. DOI: 10.1016/j. ejvs.2004.01.028

3. Moll FL, Powell JT, Fraedrich G, Verzini F, Haulon S, Waltham M, et al. Management of abdominal aortic aneurysms. Clinical Practice Guidelines of the European Society for Vascular Surgery. Eur J Vasc Endovasc Surg. 2011;41,S1eS58. DOI: 10.1016/j. ejvs.2010.09.011

4. Long A, Rouet L, Lindholt JS, Allaire E. Measuring the maximum diameter of native abdominal aortic aneurysms: Review and critical analysis. Eur J Vasc Endovasc Surg. 2012;43:515-524. DOI: 10.1016/j. ejvs.2012.01.018

5. Mora C, Marcus C, Barbe C, Ecarnot F, Long A. Measurement of maximum diameter of native abdominal aortic aneurysm by angio-CT: Reproducibility is better with the semi-automated method. Eur J Vasc Endovasc Surg. 2014;47:139-150. DOI: 10.1016/j. ejvs.2013.10.013

6. Bland JM, Altman DG. Statistical methods for assessing agreement between two methods of clinical measurement. Lancet. 1986;1:307-310. 10.1016/S01406736(86)90837-8

7. Jaakkola $P$, Hippeläinen $M$, Farin $P$, Rytkönen $H$, Kainulainen $H$, Partanen $K$. Interobserver variability in measuring the dimensions of the abdominal aorta: Comparison of ultrasound and computed tomography. Eur J Vasc Endovasc Surg. 1996;12:230-237. DOI: 10.1016/S10785884(96)80112-2

8. Sprouse LR, Meier GH, Parent FN, DeMasi $\mathrm{RJ}$, Glickman MH, Barber GA. Is ultrasound more accurate than computed tomography for determination of maximal abdominal aortic aneurysm? Eur J Vasc Endovasc Surg. 2004;28:28-35. DOI: 10.1016/j. ejvs.2004.03.022

9. Cayne NS, Veith FJ, Lipsitz EC, Ohki T, Mehta $M$, Gargiulo N, et al. Variability of maximal aortic aneurysm diameter measurements on CT scan: Significance and methods to minimize. J Vasc Surg. 2004;39:811-815. DOI: 10.1016/j.jvs.2003.11.042

10. England A, Niker A, Redmond C. Variability of vascular CT measurement techniques used in the assessment abdominal aortic aneurysms. Radiography. 2010;16:173181. DOI: $10.1016 /$ j.radi.2010.02.005

11. Kauffmann C, Tang A, Dugas A, Therasse É, Oliva V, Soulez G. Clinical validation of a software for quantitative follow-up of abdominal aortic aneurysm maximal diameter and growth by CT angiography. Eur J Radiol. 2011;77:502-508. DOI: 10.1016/j. ejrad.2009.07.027

12. Dugas A, Therasse E, Kauffmann C, Tang A, Elkouri S, Nozza A, et al. Reproducibility of 
abdominal aortic aneurysm diameter measurement and growth evaluation on axial and multiplanar computed tomography reformations. Cardiovasc Intervent Radiol. 2012;35:779-787. DOI: 10.1007/s00270011-0259-y

13. Paravastu SC, Jayarajasingam $R$, Cottam $R$, Palfreyman SJ, Michaels JA, Thomas SM. Endovascular repair of abdominal aortic aneurysm. Cochrane Database Syst Rev. 2014 Jan 23;1:CD004178. [Epub ahead of print] DOI: 10.1002/14651858.cd004178. pub2

14. RESCAN Collaborators, Bown MJ, Sweeting MJ, Brown LC, Powell JT, Thompson SG. Surveillance intervals for small abdominal aortic aneurysms: A meta-analysis. JAMA. 2013;309:806-813. DOI: 10.1001/ jama.2013.950

15. Investigators IT. Endovascular or open re- pair strategy for ruptured abdominal aortic aneurysm: 30 day outcomes from IMPROVE randomised trial. BMJ. 2014;348:f7661. DOI: 10.1136/bmj.f7661

16. Ghatwary TM, Patterson BO, Karthikesalingam A, Hinchliffe RJ, Loftus IM, Morgan $R$, et al. A systematic review of protocols for the three-dimensional morphologic assessment of abdominal aortic aneurysms using computed tomographic angiography. Cardiovasc Intervent Radiol. 2013;36:14-24. DOI: 10.1007/s00270-0110296-6

17. Kauffmann C, Tang A, Therasse E, Giroux MF, Elkouri S, Melanson P, et al. Measurements and detection of abdominal aortic aneurysm growth: Accuracy and reproducibility of a segmentation software. Eur J Radiol. 2012;81:1688-1694. DOI: 10.1016/j. ejrad.2011.04.044
18. Bredahl K, Taudorf $M$, Long A, Lönn L, Rouet $L$, Ardon $R$, et al. Three-dimensional ultrasound improves the accuracy of diameter measurement of the residual sac in EVAR patients. Eur J Vasc Endovasc Surg. 2013;46:525-532. DOI: 10.1016/j. ejvs.2013.09.012

Cite this article as: Mora $C$, Marcus $C$, Barbe $C$, Ecarnot F, Long A. Maximum Diameter of Native AAA Measured by Angio-CT: Reproducibility and Lack of Consensus Impacts on Clinical Decisions. AORTA 2015;3(2):47-55. DOI: $\quad$ http://dx.doi.org/10.12945/j. aorta.2015.14-059

\section{EDITOR'S COMMENTS AND QUESTIONS}

\section{Comments}

Mora and colleagues provide interesting, important information on the technique of aortic size measurement and the variations in different measurements. This work is highly important, as so many of our decisions in aortic diseases are based on size. The Editors have some questions for the authors.

\section{Questions}

1. You decided to include the wall in your diameter measurement. How do you identify the wall when contrast is used, opacifying the lumen only? The case you illustrate has a highly calcified rim, facilitating identification of the wall. What do you do if it is not calcified?

CT offers a good resolution in any direction, and in most cases interface between peri-arterial tissues (fat) and $A A A$ walls is easy to find, even if the walls are not calcified. In a few cases, if adenopathies, inferior vena cava, duodenum, small bowel are close to the aortic wall, the wall may be more difficult to identify and this requires a meticulous analysis of the slice.
2. Is it intuitively clear that the measurement with the least variability is the "true" one, as you state? Could not a method be consistent yet inaccurate? Please comment.

Unfortunately, we do not know in fact what the real maximum diameter is. Maximum diameter has to be measured on a cross-section perpendicular to the centerline. Lumen centerline is the more frequently used with angioCT but has drawbacks in case of tortuous $A A A$ with asymmetric thrombus. The AAA-wall centerline provides real cross-section perpendicular to the $A A A$, however softwares are less widespread.

3. Are there not very current commercially available programs, which identify the center line without the reader's needing to mark two center line points?

There are current commercially available programs which identify the center line without the reader's needing to mark two center line points. However placing two points on the celiac aortic level and the aortic bifurcation limits the volume of the data to analyze. 